\title{
Características produtivas e morfofisiológicas de cultivares de azevém ${ }^{1}$
}

\author{
Lucas Vargas Oliveira ${ }^{2}$, Otoniel Geter Lauz Ferreira ${ }^{2}$, \\ Régis Antonio Teixeira Coelho², Pâmela Peres Farias², Roberta Farias Silveira²
}

\begin{abstract}
Productive and morpho-physiological traits of ryegrass cultivars

In recent years, various ryegrass cultivars have been released in the Brazilian market, but it is necessary to make a proper characterization of their productive and morpho-physiological traits, in order to support the choice of the most appropriate genotype for each environment and production system. This study aimed to evaluate, under greenhouse conditions, the productive and morphophysiological traits of ryegrass cultivars, under pruning. Treatments consisted of three diploid [Comum-RS (Lolium multiflorum), Pronto $^{\circledR}$ (Lolium multiflorum var. westerwoldicum) and Conquest ${ }^{\mathbb{B}}$ (Lolium multiflorum var. Italicum)] and four tetraploid cultivars [INIA $\operatorname{Titan}^{\circledR}$ (Lolium multiflorum var. Italicum), Winter Star ${ }^{\circledR}$ (Lolium multiflorum var. westerwoldicum), KLM $138^{\circledR}$ (Lolium multiflorum var. italicum) and Banquet $\mathrm{II}^{\circledR}$ (Lolium perenne)], in a completely randomized design, totaling seven treatments, with six replications. The variables evaluated were forage weight, absolute growth rate (AGR), leaf area (LA), leaf area ratio (LAR), leaf weight ratio (LWR), specific leaf area (SLA), net assimilation rate (NAR), relative growth rate (RGR), time for the first pruning and pruning intervals. Significant differences were observed for all variables. The INIA Titan ${ }^{\circledR}$ cultivar showed the highest forage weight, while the LAR, LWR, SLA, NRA and RGR were higher for the Pronto ${ }^{\circledR}$ cultivar and the AGR was higher for the Banquet II ${ }^{\circledR}$ cultivar. The Pronto ${ }^{\circledR}$ cultivar presented early yield ( 51 days until the first pruning) and fast regrowth (11 days for pruning interval) and the INIA Titan ${ }^{\circledR}$ rapid regrowth (15 days for pruning interval) combined with high forage weight.
\end{abstract}

KEY-WORDS: Lolium multiflorum; Lolium perenne; yield and regrowth rate.

\section{INTRODUÇÃO}

O azevém é a planta forrageira de inverno mais utilizada no Rio Grande do Sul, assim como na maior parte das regiões temperadas e subtropicais do globo (Bressolin 2007). As duas principais

\section{RESUMO}

Nos últimos anos, diversas cultivares de azevém têm sido lançadas no mercado brasileiro, sendo necessário que estejam devidamente caracterizadas produtiva e morfofisiologicamente, para subsidiar a escolha do genótipo adequado a cada ambiente e sistema de produção. Assim, objetivou-se avaliar, em casade-vegetação, as características produtivas e morfofisiológicas de cultivares de azevém submetido a cortes. Os tratamentos consistiram de três cultivares diploides [Comum-RS (Lolium multiflorum), Pronto ${ }^{\circledR}$ (Lolium multiflorum var. westerwoldicum) e Conquest $^{\circledR}$ (Lolium multiflorum var. italicum)] e quatro tetraploides [INIA Titan ${ }^{\circledR}$ (Lolium multiflorum var. italicum), Winter $\operatorname{Star}^{\circledR}$ (Lolium multiflorum var. westerwoldicum), KLM $138^{\circledR}$ (Lolium multiflorum var. italicum) e Banquet II ${ }^{\circledR}$ (Lolium perenne)], dispostas em delineamento inteiramente casualizado, constituindo sete tratamentos, com seis repetições. Foram analisadas a massa de forragem, taxa de crescimento absoluto (TCA), área foliar (AF), razão de área foliar (RAF), razão de massa de folhas (RMF), área foliar específica (AFE), taxa de assimilação líquida (TAL), taxa de crescimento relativo (TCR), tempo para o primeiro corte e intervalo de cortes. Houve diferença para todas as variáveis citadas. Maior massa de forragem foi observada para a cultivar INIA Titan ${ }^{\circledR}$, bem como maiores RAF, RMF, AFE, TAL e TCR, para a cultivar Pronto $^{\circledR}$, e maior TCA, para Banquet II $^{\circledR}$. A cultivar Pronto ${ }^{\circledR}$ apresentou produção precoce (51 dias até o primeiro corte) e rápido rebrote (11 dias de intervalo de corte) e a INIA Titan ${ }^{\circledR}$ rápido rebrote (15 dias de intervalo de corte), aliado a elevada massa de forragem.

PALAVRAS-CHAVE: Lolium multiflorum; Lolium perenne; índice de produção e rebrote.

espécies do gênero Lolium, L. multiflorum (azevém anual) e L. perenne (azevém perene), cruzam livremente entre si e, como resultado da evolução e adaptação natural a distintos ambientes, assim como dos cruzamentos realizados por melhoristas, apresentam gradiente de variação contínua, que vai

1. Trabalho recebido em jun./2013 e aceito para publicação em jun./2014 ( ${ }^{\circ}$ registro: PAT 24497).

2. Universidade Federal de Pelotas (UFPel), Faculdade de Agronomia Eliseu Maciel, Pelotas, RS, Brasil.

E-mails: lvoliveira.agro@gmail.com,oglferreira@gmail.com,regisnz_57@hotmail.com, pamperesf@hotmail.com, robertafarias@zootecnista.com.br. 
desde formas estritamente anuais a perenes (INIA 2010).

O azevém é uma gramínea que pode possuir dois níveis de ploidia $(2 \mathrm{n}=2 \mathrm{x}=14$ cromossomos ou $2 \mathrm{n}=4 \mathrm{x}=28$ cromossomos), determinando diferentes características genotípicas e fenotípicas. A duplicação de cromossomos afeta, diretamente, o desempenho da planta, devido ao aumento no volume celular (Balocchi \& López 2009). Com isto, o teor de água se eleva, assim como os teores de carboidratos solúveis, proteínas e lipídios, aumentando a digestibilidade, eficiência ruminal e desempenho animal (Smith et al. 2001, Nair 2004).

Conforme Rouquette Junior \& Nelson (1997) e Pereira et al. (2012), a produção do autotetraploide objetiva aumentar caracteres de interesse agronômico, como qualidade e massa de forragem, resistência a doenças, uniformidade e estabilidade das populações. As cultivares tetraploides também se diferenciam das diploides por apresentarem folhas mais largas e de coloração mais escura, menor número de perfilhos de maior tamanho, ciclo vegetativo mais longo, maior precocidade, menor tolerância ao frio e ao estresse hídrico e maior exigência em fertilidade do solo, para expressar seu potencial de crescimento (Freitas 2003, Blount et al. 2005, Sugiyama 2006).

Nos últimos anos, diversas cultivares de azevém têm sido lançadas no mercado brasileiro, aumentando as possibilidades de escolha do genótipo adequado a cada ambiente e sistema de produção. Entretanto, o conhecimento de muitas características fisiológicas e de crescimento destas cultivares ainda é incipiente, ou mesmo inexistente, fazendo com que, muitas vezes, sua indicação de uso seja baseada em técnicas empíricas.

De acordo com Oliveira et al. (2000), cultivares de uma mesma espécie podem apresentar comportamentos diferenciados, com relação aos índices de crescimento. Neste sentido, Lawlor (1995) comenta que, frequentemente, para determinada condição de meio, a massa de forragem mostra melhor correlação com características da planta (como a razão de área foliar) ou do relvado (índice de área foliar), associadas à intercepção de luz, do que com a sua eficiência fotossintética.

O balanço fotossíntese-respiração determina a quantidade de assimilado disponível para o crescimento vegetal, sendo denominado taxa de assimilação líquida. Entretanto, a alocação preferencial de carbono, pelas diferentes frações da planta (folhas, colmo e sistema radicular), condicionada pelo meio e/ou manejo, determina variações nos índices de crescimento, como a área foliar específica, razão de peso de folha e razão de área foliar (Gomide \& Gomide 1999).

Do ponto de vista agronômico, as análises dos índices de crescimento servem para conhecer diferenças funcionais e estruturais entre cultivares de uma mesma espécie, de modo a selecioná-las dentro de programas de melhoramento genético (Benincasa 2003).

Objetivou-se, com este estudo, avaliar as características produtivas e morfofisiológicas de cultivares de azevém submetido a cortes.

\section{MATERIAL E MÉTODOS}

O experimento foi conduzido em casa-de-

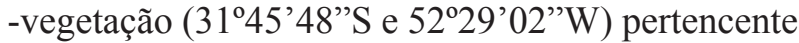
à Faculdade de Agronomia Eliseu Maciel da Universidade Federal de Pelotas, Campus Capão do Leão (RS), em um período experimental de 225 dias (06/06/2011 a 17/01/2012).

Os tratamentos consistiram de três cultivares diploides [Comum-RS (Lolium multiflorum), Pronto $^{\circledR}$ (Lolium multiflorum var. westerwoldicum) e Conquest $^{\circledR}$ (Lolium multiflorum var. italicum)] e quatro tetraploides [INIA Titan ${ }^{\circledR}$ (Lolium multiflorum var. italicum), Winter $\operatorname{Star}^{\circledR}$ (Lolium multiflorum var. westerwoldicum), KLM $138^{\circledR}$ (Lolium multiflorum var. italicum) e Banquet $\mathrm{II}^{\circledR}$ (Lolium perenne)], que foram semeadas em 06/06/2011, na densidade de 10 sementes vaso ${ }^{-1}$ contendo $2,5 \mathrm{~kg}$ de solo, com as seguintes características químicas: $\mathrm{pH}\left(\mathrm{H}_{2} \mathrm{O}\right)=5$; índice $\mathrm{SMP}=6,2$; matéria orgânica $=1,8 \%$; argila $=$ $20 \%$; CTC $=8 \mathrm{cmol}_{\mathrm{c}} \mathrm{dm}^{-3} ; \mathrm{P}=12,5 \mathrm{mg} \mathrm{dm}^{-3} ;$ e K $=$ $62 \mathrm{mg} \mathrm{dm}^{-3}$. O solo foi corrigido com 4,62 g de calcário e adubado em dose única, antes da semeadura, com 0,137 g da fórmula 10-30-10 vaso-1 (SBCS 2004). Foram utilizados seis vasos por cultivar, mantidos sem restrição hídrica, constituindo um experimento em delineamento inteiramente casualizado, com sete tratamentos e seis repetições.

A partir do aparecimento da primeira folha completamente expandida, foi realizado o raleio das plantas, mantendo-se três por vaso. Quando as plantas atingiram $20 \mathrm{~cm}$ de altura média, foi realizado o primeiro corte, com resíduo de $7,0 \mathrm{~cm}$, para dar condições ao melhor estabelecimento das mesmas (Freitas 2003, Pedroso et al. 2005). Os 
demais cortes foram realizados quando as plantas atingiram $15 \mathrm{~cm}$ de altura, mantendo-se o mesmo resíduo $(7,0 \mathrm{~cm})$ do primeiro corte (Medeiros \& Nabinger 2001).

Após o corte e separação das frações botânicas folha e caule, o material foi colocado em estufa de ventilação forçada e seco a $65^{\circ} \mathrm{C}$, por 72 horas, para a determinação do teor de matéria seca e cálculo do somatório da massa de forragem $\left(\mathrm{g}_{\text {vaso }}{ }^{-1}\right)$ obtida nos cortes. A determinação da área das folhas das três plantas presentes em cada vaso foi realizada por meio de imagens digitalizadas das mesmas, utilizando-se o software Determinador Digital de Áreas - DDA (Ferreira et al. 2012).

A partir desses resultados, foram calculadas, conforme Benincasa (2003), a razão de área foliar - RAF (área foliar/MS total); área foliar específica - AFE (área foliar/MS folha); razão de massa de folhas - RMF (MS folha/MS total); taxa de crescimento absoluto - TCA (massa de forragem acumulada entre dois cortes consecutivos/intervalo de tempo entre os cortes); taxa de assimilação líquida - TAL \{TCA x [Ln (área foliar)/área foliar $]$; e taxa de crescimento relativo - TCR (RAF x TAL).

As variáveis fisiológicas citadas anteriormente foram determinadas a cada corte, calculando-se, posteriormente, a média dos cortes. A soma térmica diária acumulada foi calculada por meio da seguinte equação: graus dia $(\mathrm{GD})=[(\mathrm{Tmax}+\mathrm{TMin}) / 2]-\mathrm{Tb}$, em que Tmax $=$ temperatura máxima, $T$ min $=$ temperatura mínima e $\mathrm{Tb}=$ temperatura de base. $\mathrm{A} \mathrm{Tb}$ foi considerada como $7^{\circ} \mathrm{C}$ e $9^{\circ} \mathrm{C}$, respectivamente para azevéns diploides e tetraploides (Müller et al. 2009). Os valores de temperatura do ar foram coletados no local do experimento, utilizando-se termômetro de máxima e mínima. Em 05/10/2011, 04/11/2011 e 10/11/2011, as cultivares Comum-RS, Pronto ${ }^{\circledR}$ e Winter Star $^{\circledR}$ entraram em estádio reprodutivo, não sendo mais avaliadas. As demais permaneceram em estádio vegetativo até o encerramento do experimento (17/01/2012).

Os dados foram submetidos à análise de variância e comparados pelo teste Tukey $(\mathrm{p}<0,05)$, utilizando-se o pacote estatístico SAS (SAS Institute Inc. 1999).

\section{RESULTADOS E DISCUSSÃO}

A massa de forragem foi diferente $(p<0,0001)$ entre as cultivares, observando-se, respectivamente, $o$ maior e menor valor para as cultivares INIA $\operatorname{Titan}^{\circledR} \mathrm{e}$ Banquet II ${ }^{\circledR}$ (Tabela 1), não havendo clara diferenciação entre a massa de forragem obtida pelas cultivares diploides e tetraploides. A superioridade da primeira cultivar está associada ao seu rápido estabelecimento, ciclo longo e curto intervalo de cortes (Tabela 1).

A cada acúmulo de 215,87 graus dia (GD), que, no presente estudo, ocorreu, aproximadamente, a cada 15 dias, foi possível realizar um corte, permitindo a execução de 11 cortes. A cultivar INIA $\operatorname{Titan}^{\circledR}$, juntamente com a Conquest ${ }^{\circledR}$, apresentou o segundo menor período de tempo até o primeiro corte, evidenciando, assim, da mesma forma que para a cultivar Pronto ${ }^{\circledR}$, que apresentou o menor período para o primeiro corte, a possibilidade da sua utilização precoce.

Maior massa de forragem de INIA Titan ${ }^{\circledR}$, em relação a outros genótipos, também foi relatada por Rocha et al. (2007), que compararam a INIA Titan ${ }^{\circledR}$ com a Estanzuela 284, Cetus e algumas cultivares de

Tabela 1. Massa de forragem (soma dos cortes), taxa de crescimento absoluto médio entre os cortes e características produtivas de cultivares diploides e tetraploides de azevém (Capão do Leão, RS, 2011/2012).

\begin{tabular}{|c|c|c|c|c|c|c|c|c|c|}
\hline \multirow{2}{*}{$\begin{array}{l}\text { Nível de } \\
\text { ploidia }\end{array}$} & \multirow{2}{*}{ Cultivar } & \multirow{2}{*}{$\begin{array}{l}\mathrm{N}^{\mathrm{o}} \text { de } \\
\text { cortes }\end{array}$} & \multicolumn{2}{|c|}{ Intervalo de cortes } & \multirow{2}{*}{$\begin{array}{c}\mathrm{N}^{\mathrm{o}} \text { de dias até } \\
\text { o } 1^{\mathrm{o}} \text { corte }\end{array}$} & \multirow{2}{*}{$\begin{array}{l}\mathrm{N}^{0} \text { de dias até } \\
\mathrm{o} \text { último corte }\end{array}$} & \multirow{2}{*}{$\begin{array}{l}\text { Massa de } \\
\text { Forragem } \\
\left(\mathrm{g} \mathrm{vaso}^{-1}\right)\end{array}$} & \multicolumn{2}{|c|}{$\begin{array}{c}\text { Taxa de crescimento } \\
\text { absoluto }\end{array}$} \\
\hline & & & Dias & GD & & & & $\left(\mathrm{g} \mathrm{dia}^{-1}\right)$ & $\left(\mathrm{g} \mathrm{GD}^{-1}\right)$ \\
\hline \multirow{3}{*}{ Diploide } & Comum-RS & $4 \mathrm{c}$ & $13 \mathrm{e}$ & $274,42 \mathrm{e}$ & $82 \mathrm{~b}$ & $121 \mathrm{e}$ & $3,43 \mathrm{bc}$ & $0,028 \mathrm{~b}$ & $0,0125 \mathrm{~d}$ \\
\hline & Conquest $^{\circledR}$ & $5 \mathrm{bc}$ & $38 \mathrm{a}$ & $652,75 \mathrm{a}$ & $72 \mathrm{~d}$ & $224 \mathrm{a}$ & $3,20 \mathrm{bc}$ & $0,014 \mathrm{f}$ & $0,0049 \mathrm{~g}$ \\
\hline & Pronto $^{\circledR}$ & $10 \mathrm{a}$ & $11 \mathrm{f}$ & $140,53 \mathrm{~g}$ & $51 \mathrm{e}$ & $150 \mathrm{~d}$ & $3,11 \mathrm{bc}$ & $0,021 \mathrm{~d}$ & $0,02213 \mathrm{a}$ \\
\hline \multirow{4}{*}{ Tetraploide } & INIA Titan $^{\circledR}$ & $11 \mathrm{a}$ & $15 \mathrm{~d}$ & $215,87 \mathrm{f}$ & $72 \mathrm{~d}$ & $222 b$ & $4,13 \mathrm{a}$ & $0,019 \mathrm{e}$ & $0,01913 \mathrm{~b}$ \\
\hline & Winter Star ${ }^{\circledR}$ & $5 \mathrm{bc}$ & $17 \mathrm{c}$ & $277,00 \mathrm{~d}$ & $88 \mathrm{a}$ & $156 \mathrm{c}$ & $3,65 \mathrm{abc}$ & $0,023 \mathrm{c}$ & $0,01317 \mathrm{c}$ \\
\hline & KLM $138^{\circledR}$ & $6 \mathrm{~b}$ & $30 \mathrm{~b}$ & $448,25 \mathrm{c}$ & $75 \mathrm{c}$ & $225 \mathrm{a}$ & $3,69 \mathrm{ab}$ & $0,019 \mathrm{e}$ & $0,00823 \mathrm{e}$ \\
\hline & Banquet II ${ }^{\circledR}$ & $2 \mathrm{~d}$ & $12 \mathrm{ef}$ & $464,65 \mathrm{~b}$ & $88 \mathrm{a}$ & $100 \mathrm{f}$ & $3,05 \mathrm{c}$ & $0,030 \mathrm{a}$ & $0,00656 \mathrm{f}$ \\
\hline CV (\%) & & 13,29 & 4,2 & 0,23 & 1,08 & 0,48 & 9,88 & 3,71 & 0,65 \\
\hline
\end{tabular}

Médias seguidas da mesma letra, na coluna, não diferem, significativamente, entre si, pelo teste Tukey $(\alpha=0,05)$. GD: graus dia. 
aveia, utilizando, como critério de corte, a presença de três folhas completamente expandidas.

A cultivar Banquet $\mathrm{II}^{\circledR}$, mesmo apresentando menor massa de forragem, mostrou maior taxa de crescimento absoluto (Tabela 1). Embora esta cultivar tenha necessitado de longo período ( 88 dias) para o primeiro corte, em função da altura preconizada para este, o segundo se deu 12 dias após, resultando em acúmulo de 0,00656 g a cada GD (ou 0,03 g a cada dia de crescimento), o que indica velocidade média de crescimento, ao longo do período de observação, superior às demais. Como a cultivar, em razão do seu hábito prostrado de crescimento, não atingiu mais a altura para corte até o final do experimento, sofreu apenas dois cortes, permanecendo, porém, em virtude de ser perene, com perfilhos em estádio vegetativo. As demais cultivares apresentaram massa de forragem dentro da amplitude delimitada pelos extremos já citados (Tabela 1).

As altas somas térmicas entre os cortes, apresentadas por algumas das cultivares do tipo italiano, se deram em função das temperaturas médias ocorridas no interior da casa-de-vegetação, nos meses de novembro $\left(24,3^{\circ} \mathrm{C}\right)$, dezembro $\left(25,4^{\circ} \mathrm{C}\right)$ e janeiro $\left(25,9^{\circ} \mathrm{C}\right)$. Como estas extrapolaram a temperatura máxima para crescimento do azevém $\left(25^{\circ} \mathrm{C}\right.$; Rouquette Junior \& Nelson 1997), provavelmente, em alguns dias, houve paralisação do crescimento das plantas, fato que, apesar de ter proporcionado aumento no intervalo de cortes, demonstrou uma possível tolerância destas cultivares às elevadas temperaturas que, frequentemente, ocorrem no verão. As cultivares do tipo westerwoldicum, por sua vez, por serem de ciclo mais curto (Rouquette Junior \& Nelson 1997), entraram em estádio reprodutivo antes da ocorrên- cia destas temperaturas, não sendo avaliadas após a ocorrência do evento.

Apesar das diferenças observadas entre as cultivares, nas variáveis fisiológicas, não foram detectadas características diferenciais entre diploides e tetraploides. As cultivares apresentaram resultados de razão de área foliar (RAF) diferentes $(\mathrm{p}<0,0001)$, sendo observado maior valor para a cultivar Pronto ${ }^{\circledR}$ (Tabela 2). A RAF representa a quantidade de área foliar usada pela planta para produzir uma unidade de massa de matéria seca (Benincasa 2003). Seu alto valor demonstra maior alocação de assimilados para o desenvolvimento de folhas, nesta cultivar, em relação às demais, o que se refletiu no grande número de cortes da mesma. Embora sua área foliar por corte tenha sido baixa, o resultado de RAF indica que esta cultivar aloca assimilados prioritariamente para a produção de folhas, em detrimento das outras estruturas vegetativas.

A baixa RAF encontrada nas cultivares Winter Star $^{\circledR}$ e Banquet $\mathrm{II}^{\circledR}$ se deve aos valores reduzidos de área foliar específica (AFE), já que a RAF é o produto da AFE pela RMF (Santos Júnior et al. 2004). Conforme Gomide (1997), a variação na AFE contribui indiretamente, via interceptação de luz, para o crescimento da planta, enquanto a variação na RMF tem efeito direto na produção de massa seca. Andrade et al. (2002) atribuem os decréscimos na RAF ao investimento de fotoassimilados para o desenvolvimento de órgãos não fotossintetizantes, como colmos, bainhas e raízes, tornando a planta mais ineficiente, já que a massa total aumenta e a superfície fotossintetizante varia pouco.

No presente estudo, apenas a cultivar Comum-RS apresentou menor valor $(\mathrm{p}<0,0001)$

Tabela 2. Área foliar (AF), razão de área foliar (RAF), razão de massa de folha (RMF) e área foliar específica (AFE) de cultivares diploides e tetraploides de azevém (média dos cortes) (Capão do Leão, RS, 2011/2012).

\begin{tabular}{llcccc}
\hline Nível de ploidia & \multicolumn{1}{c}{ Cultivar } & $\begin{array}{c}\text { AF } \\
\left(\mathrm{cm}^{2} \mathrm{vaso}^{-1}\right)\end{array}$ & $\begin{array}{c}\text { RAF } \\
\left(\mathrm{cm}^{2} \mathrm{~g}^{-1}\right)\end{array}$ & $\begin{array}{c}\text { RMF } \\
\left(\mathrm{g}^{-1}\right)\end{array}$ & $\begin{array}{c}\text { AFE } \\
\left(\mathrm{cm}^{2} \mathrm{~g}^{-1}\right)\end{array}$ \\
\hline \multirow{2}{*}{ Diploide } & Comum-RS $^{*}$ & $122,23 \mathrm{a}$ & $135,54 \mathrm{~b}$ & $0,89 \mathrm{~b}$ & $157,88 \mathrm{~b}$ \\
& Conquest $^{\circledR}$ & $85,29 \mathrm{~b}$ & $114,73 \mathrm{~b}$ & $0,98 \mathrm{a}$ & $115,07 \mathrm{c}$ \\
& Pronto $^{\circledR}$ & $54,16 \mathrm{c}$ & $223,90 \mathrm{a}$ & $0,95 \mathrm{a}$ & $226,31 \mathrm{a}$ \\
\hline \multirow{2}{*}{ Tetraploide } & INIA Titan $^{\circledR}$ & $63,60 \mathrm{c}$ & $129,99 \mathrm{~b}$ & $0,99 \mathrm{a}$ & $130,13 \mathrm{bc}$ \\
& Winter Star $^{\circledR}$ & $52,76 \mathrm{c}$ & $70,20 \mathrm{c}$ & $0,98 \mathrm{a}$ & $71,01 \mathrm{~d}$ \\
& KLM 138 $^{\circledR}$ & $90,49 \mathrm{~b}$ & $115,79 \mathrm{~b}$ & $0,99 \mathrm{a}$ & $115,95 \mathrm{c}$ \\
\hline $\mathrm{CV}(\%)$ & Banquet II $^{\circledR}$ & $122,47 \mathrm{a}$ & $77,97 \mathrm{c}$ & $1,00 \mathrm{a}$ & $77,97 \mathrm{~d}$ \\
\hline
\end{tabular}

Médias seguidas da mesma letra, na coluna, não diferem, significativamente, entre si, pelo teste Tukey $(\alpha=0,05)$. 
de RMF (Tabela 2). Esta cultivar, em virtude de apresentar um dos menores ciclos, em relação às demais (Tabela 1), alocou maior fração de material fotossintetizado para órgãos vegetais que não folhas, ocasionando significativa redução da RMF. De modo geral, as demais cultivares apresentaram alta produção de folhas, em relação a colmos, evidenciando os aspectos qualitativos dos genótipos, visto que folhas constituem a fração mais importante na alimentação de ruminantes, influenciando na digestibilidade, degradação ruminal e desempenho animal.

$\mathrm{O}$ maior valor $(\mathrm{p}<0,0001)$ de área foliar específica (AFE) foi observado na cultivar Pronto ${ }^{\circledR}$, enquanto os menores foram observados nas tetraploides (Tabela 2), com exceção da cultivar Conquest ${ }^{\mathbb{R}}$. A magnitude da AFE está diretamente relacionada com a anatomia foliar, como cutículas e epiderme mais delgadas, menor espessura de mesófilo e menor proporção de parênquima paliçádico de tecidos condutores e de sustentação, maior proporção de espaços intercelulares e menor densidade estomática (Berlyn \& Cho 2000), sendo que seu inverso indica a espessura da folha (Benincasa 2003). Conforme Kephart \& Buxton (1989), embora haja exceções, a espessura da folha, nas gramíneas, é alterada por modificações no tamanho das células, e não pela redução do seu número, enquanto o aumento da área foliar é afetado tanto pela expansão das células como pela sua divisão (Friend \& Pomeroy 1970).

Diferenças de AFE e, consequentemente, de espessura de folhas, são, frequentemente, observadas quando as plantas são submetidas a sombreamento (Garcez Neto et al. 2010, Gobbi et al. 2011). Segundo (Gobbi et al. 2011), o aumento da AFE, em folhas de Urochloa decumbens cv. Basilisk, foi acompanhado pela redução linear da espessura da folha, em função de níveis crescentes de sombra.

Normalmente, genótipos com folhas de menor espessura utilizam mais energia para a produção de pigmentos capturadores de luz, utilizando, praticamente, toda a energia luminosa incidente e aumentando sua atividade fotoquímica, o que leva a grande capacidade fotossintética das folhas (Castro \& Garcia 1996).

Conforme Salisbury \& Ross (1985), plantas que vivem em sombreamento tendem a se adaptar a esta condição ambiental, aumentando a área foliar e diminuindo a espessura das folhas, o sistema radicular e o peso seco da planta. Diferenças de AFE entre espécies também podem ocorrer como função da característica genética da planta, possivelmente denotando maior ou menor capacidade de sobrevivência, em ambientes sombreados (Benincasa 2003).

O maior valor de taxa de assimilação líquida (TAL) foi observado nas cultivares Pronto ${ }^{\circledR} \mathrm{e}$ Winter $\mathrm{Star}^{\circledR}$, que apresentaram grande quantidade de folhas em expansão e diferiram $(p<0,0001)$ das demais (Tabela 3).

Segundo Milthorpe \& Davidson (1966), folhas que ainda não concluíram sua expansão (sem visualização da lígula) não translocam assimilados para outras partes do perfilho, utilizando-os para o seu próprio desenvolvimento. Além disto, recebem assimilados provenientes das folhas completamente expandidas (Pedreira et al. 2001). Neste sentido, Oliveira et al. (2000) atribuíram os altos valores de TAL observados na fase de rebrota de Cynodon spp. à presença de folhas jovens provenientes dos rebrotes, as quais apresentam maior eficiência fotossintética.

O menor valor de TAL, observado nas cultivares Comum-RS e KLM $138^{\circledR}$, possivelmente, se deu em função da redução na eficiência fotossintética das folhas, em decorrência da avançada idade média das mesmas e consequente senescência, além do possível aumento progressivo das taxas respiratórias de manutenção (Watson et al. 1966, Woledge \& Leafe 1976, Parsons et al. 1983). Além disto, a cultivar Comum-RS entrou em estádio reprodutivo antes das demais, apresentando menor proporção de folhas, em relação aos colmos, estruturas menos eficientes, resultando em decréscimo esperado, uma vez que a área foliar útil à fotossíntese foi reduzida (Conceição et al. 2005, Pedó et al. 2010).

A TAL é uma medida da eficiência fotossintética da planta e representa o balanço entre o material

Tabela 3. Taxa de assimilação líquida (TAL) e taxa de crescimento relativo (TCR) de cultivares diploides e tetraploides de azevém (média dos cortes) (Capão do Leão, RS, 2011/2012).

\begin{tabular}{clcc}
\hline $\begin{array}{c}\text { Nível de } \\
\text { ploidia }\end{array}$ & \multicolumn{1}{c}{ Cultivar } & $\begin{array}{c}\text { TAL } \\
\left(\mathrm{g} \mathrm{cm}^{2} \mathrm{dia}^{-1}\right)\end{array}$ & $\begin{array}{c}\text { TCR } \\
\left(\mathrm{g} \mathrm{g}^{-1} \mathrm{dia}^{-1}\right)\end{array}$ \\
\hline \multirow{3}{*}{ Diploide } & Comum-RS & $0,0011 \mathrm{bc}$ & $0,1492 \mathrm{c}$ \\
& Conquest $^{\circledR}$ & $0,00073 \mathrm{~d}$ & $0,084 \mathrm{~g}$ \\
& Pronto $^{\circledR}$ & $0,00155 \mathrm{a}$ & $0,3465 \mathrm{a}$ \\
\hline \multirow{5}{*}{ Tetraploide } & INIA Titan $^{\circledR}$ & $0,00124 \mathrm{~b}$ & $0,1612 \mathrm{~b}$ \\
& Winter Star $^{\circledR}$ & $0,00173 \mathrm{a}$ & $0,12 \mathrm{~d}$ \\
& ${\text { KLM } 138^{\circledR}}^{\circledR}$ & $0,00095 \mathrm{c}$ & $0,1095 \mathrm{e}$ \\
& Banquet II $^{\circledR}$ & $0,00118 \mathrm{~b}$ & $0,0918 \mathrm{f}$ \\
\hline $\mathrm{CV}(\%)$ & & 6,73 & 4,25 \\
\hline
\end{tabular}

Médias seguidas da mesma letra, na coluna, não diferem, significativamente, entre si, pelo teste Tukey $(\alpha=0,05)$. 
produzido pela fotossíntese e o material perdido pela respiração (Silva et al. 2000). De acordo com Pilbeam (1992), indivíduos que apresentam baixa perda de carbono pela respiração devem ser preferidos em programas de seleção de novas cultivares forrageiras, pois apresentam menor custo com a respiração e, frequentemente, são mais produtivos.

Em relação à taxa de crescimento relativo (TCR), verificou-se diferença ( $\mathrm{p}<0,0001)$ entre as cultivares, observando-se maior valor para a cultivar Pronto $^{\circledR}$ (Tabela 3), ressaltando a maior dependência desta variável, com relação à RAF (Tabela 2) do que com relação à TAL (Tabela 1). Tais resultados vão ao encontro dos observados por Poorter (1989), que cita a RAF como o principal componente da TCR, determinando a sua variação. A TCR consiste no incremento da matéria seca total, em relação à matéria seca já existente (Benincasa 1988), e, conforme Evans (1972), Garnier (1992) e Pinto (1993), pode ser influenciada por fatores como espécie, variedade e estádio de desenvolvimento.

\section{CONCLUSÃO}

A cultivar Pronto ${ }^{\circledR}$ caracterizou-se como precoce, apresentando rápido rebrote e, simultaneamente, maiores valores de razão de área foliar, razão de massa de folha, área foliar específica, taxa de assimilação líquida e taxa de crescimento relativo, enquanto a INIA Titan ${ }^{\circledR}$ caracterizou-se por apresentar rápido rebrote e elevada massa de forragem.

\section{REFERÊNCIAS}

ANDRADE, A. C. et al. Análise de crescimento do capimelefante napier adubado e irrigado. In: REUNIÃO ANUAL DA SOCIEDADE BRASILEIRA DE ZOOTECNIA, 39., 2002, Recife. Anais... Recife: SBZ, 2002. 1 CD-ROM.

BALOCCHI, O. A.; LÓPEZ, I. L. Herbage production, nutritive value and grazing preference of diploid and tetraploid perennial ryegrass cultivars (Lolium perenne L.). Chilean Journal of Agricultural Research, Chillan, v. 69, n. 3, p. 331-339, 2009.

BENINCASA, M. M. P. Análise do crescimento de plantas. Jaboticabal: Funep, 1988.

BENINCASA, M. M. P. Análise de crescimento de plantas: noções básicas. Jaboticabal: Funep, 2003.

BERLYN, G. P.; CHO, J. Light, moisture, and nutrient use by plants. In: ASHTON, M. S.; MONTAGNINI, F.
(Eds.). The silvicultural basis for agroforestry systems. Boca Raton: CRC Press, 2000. p. 9-39.

BLOUNT, A. R. et al. Annual ryegrass. Tampa: University of Florida, 2005.

BRESSOLIN, A. P. S. Avaliação de populações de azevém quanto à tolerância ao alumínio tóxico e estimativa de tamanho de amostra para estudos de diversidade genética com marcadores AFLP. 2007. 76 f. Dissertação (Mestrado em Agronomia) - Universidade Federal de Pelotas, Pelotas, 2007.

CASTRO, C. R. T. de; GARCIA, R. Competição entre plantas com ênfase no recurso luz. Ciência Rural, Santa Maria, v. 26, n. 1, p. 167-174, 1996.

CONCEIÇÃO, M. K. et al. Análise de crescimento de plantas de batata-doce (Ipomoea batatas (L.) Lam) cultivares Abóbora e Da Costa. Revista Brasileira de Agrociência, Pelotas, v. 11, n. 3, p. 273-278, 2005.

EVANS, G. C. The quantitative analysis of plant growth. Oxford: Blackwell Scientific Oxford, 1972.

FERREIRA, O. G. L. et al. Measurement of the rib-eye area by the digital images method. Revista Brasileira de Zootecnia, Viçosa, v. 41, n. 3, p. 811-814, 2012.

FREITAS, T. M. S. de. Dinâmica da produção de forragem, comportamento ingestivo e produção de ovelhas Ile de France em pastagem de azevém anual (Lolium multiflorum Lam.) em resposta a doses de nitrogênio. 2003. 114 f. Dissertação (Mestrado em Zootecnia) Faculdade de Agronomia, Universidade Federal do Rio Grande do Sul, Porto Alegre, 2003.

FRIEND, D. J. C.; POMEROY, M. E. Changes in cell size and number associated with the effects of light intensity and temperature on the leaf morphology of wheat. Canadian Journal of Botany, Guelph, v. 48, n. 1, p. 85-90, 1970.

GARCEZ NETO, A. F. et al. Aclimatação morfológica de forrageiras temperadas a padrões e níveis de sombreamento. Revista Brasileira de Zootecnia, Viçosa, v. 39, n. 1, p. 4250, 2010.

GARNIER, E. Growth analysis of congeneric annual and perennial grass species. Journal of Ecology, London, v. 80, n. 4, p. 665-675, 1992.

GOBBI, K. F. et al. Área foliar específica e anatomia foliar quantitativa do capim-braquiária e do amendoimforrageiro submetidos a sombreamento. Revista Brasileira de Zootecnia, Viçosa, v. 40, n. 7, p. 1436-1444, 2011.

GOMIDE, J. A. Morfogênese e análise de crescimento de gramíneas tropicais. In: SIMPÓSIO INTERNACIONAL SOBRE PRODUÇÃO ANIMAL EM PASTEJO, 1997, Viçosa. Anais... Viçosa: UFV, 1997. p. 411-430. 
GOMidE, C. A. M.; GOMIDE, J. A. Análise de crescimento de cultivares de Panicum maximum Jacq. Revista Brasileira de Zootecnia, Viçosa, v. 28, n. 4, p. 675-680, 1999.

INSTITUTO NACIONAL DE INVESTIGACIÓN AGROPECUÁRIA (INIA). Forrajeras: catálogo de cultivares 2010. Montevideo: INIA, 2010. 1 CD-ROM.

KEPHART, K. D.; BUXTON, D. R. Adaptation and forage quality of grasses grown under shade. In: CONGRESSO INTERNACIONAL DE PASTAGENS, 16., 1989, Nice. Anais... Nice: International Grassland Society, 1989. p. 819-820.

LAWLOR, D. W. Photosynthesis, productivity and environment. Journal of Experimental Botany, Lancaster, v. 46, ed. especial, p. 1449-1461, 1995.

MEDEIROS, R. B.; NABINGER, C. Rendimento de sementes e forragem de azevém anual em resposta a dose de nitrogênio e regime de corte. Revista Brasileira de Sementes, Campinas, v. 23, n. 2, p. 145-154, 2001.

MILTHORPE, F. L.; DAVIDSON, J. L. Physiological aspects of regrowth in grasses. In: MILTHORPE, F. L.; IVINS, J. D. (Eds.). The growth of cereals and grasses. London: Butterworths, 1966. p. 241-254.

MÜLLER, L. et al. Temperatura base inferior e estacionalidade de produção de genótipos diploides e tetraploides de azevém. Ciência Rural, Santa Maria, v. 39, n. 5, p. 1343-1348, 2009.

NAIR, R. Developing tetraploid perennial ryegrass (Lolium perenne L.) populations. New Zealand Journal of Agricultural Research, Wellington, v. 47, n. 1, p. 4549, 2004.

OLIVEIRA, M. A. et al. Análise de crescimento do capimbermuda 'Tifton 85' (Cynodon spp.). Revista Brasileira de Zootecnia, Viçosa, v. 29, n. 6, p. 1930-1938, 2000.

PARSONS, A. J. et al. The physiology of grass production under grazing: I. Characteristics of leaf and canopy photosynthesis of continuously grazed sward. Journal of Applied Ecology, London, v. 20, n. 1, p. 117-126, 1983.

PEDREIRA, C. G. S. et al. O processo de produção de forragem em pastagens. In: MATTOS, W. R. S. et al. (Eds.). A produção animal na visão dos brasileiros. Piracicaba: Fealq, 2001. p. 772-807.

PEDROSO, C. E. S. et al. Preferência de ovinos sob pastejo em azevém anual em diferentes alturas. In: REUNIÃO ANUAL DA SOCIEDADE BRASILEIRA DE ZOOTECNIA, 42., 2005, Goiânia. Anais... Goiânia: Ed. da UFG, 2005. 1 CD-ROM.

PEDÓ, T. et al. Crescimento de três cultivares de rabanete (Raphanus sativus) ao longo da ontogenia das plantas. Tecnologia \& Ciência Agropecuária, João Pessoa, v. 4, n. 3, p. 23-28, 2010.

PEREIRA, R. C. et al. Duplicação cromossômica de gramíneas forrageiras: uma alternativa para programas de melhoramento genético. Ciência Rural, Santa Maria, v. 42, n. 7, p. 1278-1285, 2012.
PILBEAM, C. J. Effect of nitrogen supply on the growth and senescence of leaves of Lolium perenne with contrasting rates of leaf respiration. Annals of Botany, Oxford, v. 70, n. 4, p. 365-370, 1992.

PINTO, J. C. Crescimento e desenvolvimento de Andropogon gayanus Kunth, Panicum maximum Jack. e Setaria anceps Stapf ex Massey cultivadas em vasos, sob diferentes doses de nitrogênio. 1993. 149 f. Tese (Doutorado em Zootecnia) - Universidade Federal de Viçosa, Viçosa, 1993.

POORTER, H. Interspecific variation in relative growth rate on ecological causes and physiological consequences. In: LAMBERS, H. et al. Causes and consequences of variation in growth rate and productivity of higher plants. Hangue: SBP Academic Publishing, 1989. p. 45-67.

ROCHA, M. G. et al. Avaliação de espécies forrageiras de inverno na Depressão Central do Rio Grande do Sul. Revista Brasileira de Zootecnia, Viçosa, v. 36, n. 6, p. 1990-1999, 2007.

ROUQUETTE JUNIOR, F. M.; NELSON, L. R. Ecology, production, and management of Lolium for forage in the USA. Madison: Crop Science Society of America, 1997.

SALISBURY, F. B.; BOSS, C. W. Plant physiology. 3. ed. Belmont: Wadsworth, 1985.

SANTOS JÚNIOR, J. D. et al. Análise de crescimento do capim-marandu submetido a doses de nitrogênio. Revista Brasileira de Zootecnia, Viçosa, v. 33, n. 6, p. 1985-1991, 2004.

SAS INSTITUTE INC. SAS software. Version 9.1. Cary: SAS Institute Inc., 1999.

SILVA, L. C.; BELTRÃO, N. E. de M.; AMORIM NETO, M. da S. Análise de crescimento de comunidades vegetais. Campina Grande: Embrapa-CNAPA, 2000. (Circular técnica, 34).

SMITH, K. et al. The effects of ploidy and a phenotype conferring a high water soluble carbohydrate concentration on carbohydrate accumulation, nutritive value and morphology of perennial ryegrass (Lolium perenne). Journal of Agricultural Science, Cambridge, v. 136, n. 1, p. 65-74, 2001.

SOCIEDADE BRASILEIRA DE CIÊNCIA DO SOLO (SBCS). Comissão de Química e Fertilidade do Solo RS/ SC. Manual de adubação e calagem para Estados do Rio Grande do Sul e Santa Catarina. 10. ed. Porto Alegre: SBCS/NRS, 2004.

SUGIYAMA, S. Responses of shoot growth and survival to water stress gradient in diploid and tetraploid populations of Lolium multiflorum and L. perenne. Grasslands Science, Malden, v. 52, n. 4, p. 155-160, 2006.

WATSON, D. J. et al. Changes with age in the photosynthetic and respiratory components of the net assimilation rates of sugar beet and wheat. New Phytologist, Lancaster, v. 65, n. 4, p. 500-508, 1966.

WOLEDGE, J.; LEAFE, E. L. Single leaf and canopy photosynthesis in a ryegrass sward. Annals of Botany, Oxford, v. 40, n. 68, p. 773-783, 1976. 